LPENSL-TH 05/2000

\title{
Spin accumulation in the semi classical and quantum regimes
}

\author{
R. Mélin ${ }^{(1,2)}$ and D. Denaro ${ }^{(2)}$ \\ (1) Centre de Recherches sur les Très basses températures (CRTBT)* \\ CNRS, BP 166X, 38042 Grenoble Cedex, France \\ (2) Laboratoire de Physique ${ }^{\dagger}$ Ecole Normale Supérieure de Lyon \\ 46 Allée d'Italie, 69364 Lyon Cedex 07, France
}

\begin{abstract}
We consider spin accumulation at a ferromagnet-normal metal interface in the presence of magnetic scattering in the normal metal. In the classical regime, we discuss the inverse Drude scaling of the conductance as a function of the interface transparencies. We present a treatment based on an exact solution of the Boltzmann equation. In the quantum regime, we solve a single impurity "spin-flip Fabry Perot interferometer" for quantum coherent multiple scatterings, in which we find a resonance in the spin flip channels. This resonance appears to be the quantum analog of the semi classical inverse Drude scaling of the conductance.
\end{abstract}

\footnotetext{
${ }^{*}$ U.P.R. $5001 \mathrm{du}$ CNRS, Laboratoire conventionné avec l'Université Joseph Fourier

${ }^{\dagger}$ U.M.R. CNRS 5672
} 


\section{Introduction}

The discovery of the Giant Magneto Resistance (GMR) in magnetic multilayers [1, 2, 3] has generated an important interest. These systems are made of a sandwich of alternating ferromagnetic and non magnetic layers. Valet and Fert proposed a semi classical description of the perpendicular GMR, on the basis of a Boltzmann equation incorporating a spin-dependent transport in the presence of spin accumulation [- [s] (see also [5]). Spin accumulation occurs in the GMR because the current arising from a ferromagnet is spin polarized, and therefore cannot penetrate a ferromagnet with an opposite magnetization. Instead, spin accumulates at the interface. This phenomenon occurs also at the interface between a ferromagnet and a superconductor, where a spin polarized current cannot penetrate the superconductor [6, 7, 8]. Here, we would like to reconsider two particular aspects of spin accumulation, namely, (i) in the semi classical regime, the possibility of an inverse Drude scaling of the conductance meaning that, in some parameter range, the conductance increases with the length of the conductor; and (ii) in the quantum coherent regime, the existence of a resonance in the spin flip channels. More precisely, we study a ferromagnet - normal metal - ferromagnet spin valve, in which we assume the presence of magnetic scattering in the normal metal [9, 10]. The inverse Drude scaling resulting from spin accumulation is already implicitly contained in the equations obtained by Valet and Fert [⿶] , but, to our knowledge, this effect has not been studied previously per se in the literature, which we do here. Spin accumulation corresponds to the presence of a different chemical potential for the spin-up and spin-down electrons, which obey a spin diffusion equation [4, 11, 12]. Our treatment is not based on the spin diffusion equation, but relies on an exact solution of the 1D Boltzmann equation where we can make an exact decoupling between the charge and spin sectors.

Next, we ask to what extend a quantum model can show a similar physics. We are lead to study a quantum "spin-flip Fabry Perot" interferometer in which a single magnetic impurity is located at a

given distance $a$ away from a ferromagnet interface. We find the existence of a Fabry-Perot resonance in the spin-flip channels as the parameter $a$ is varied. This resonance disappears as the ferromagnet spin polarization is decreased, and can therefore be viewed as the equivalent of the inverse Drude behavior in the quantum coherent regime. Our treatment is based on a Landauer approach, similar to the one used by Zhu and Wang to study the effect of magnetic scattering close to a superconductor interface [13].

The article is organized as follows. Section 2 is devoted to the solution of the semi classical transport equations. We solve the "spin-flip Fabry Perot" interferometer model in section 3. Final remarks are given in the Conclusion.

\section{Transport in the semi classical regime}




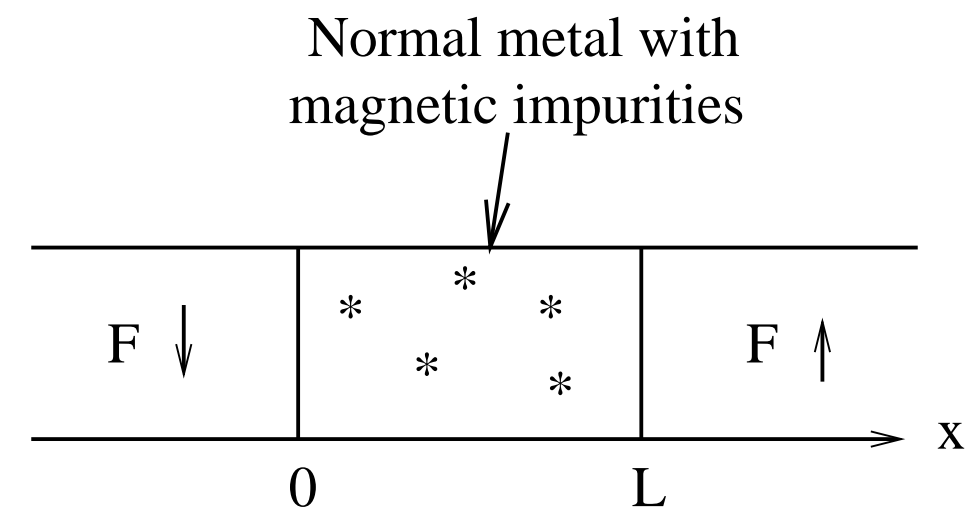

Figure 1: The "spin valve" geometry considered in section 2, consisting of a normal metal wire doped with magnetic impurities, connected to two ferromagnets with an opposite magnetization.

\subsection{Boltzmann equation and boundary conditions}

We consider a model in which magnetic impurities are present in a normal metal close to a ferromagnet interface (see Fig. 11). We neglect any Kondo correlation [14], which is an assumption valid above the Kondo temperature. The presence of the ferromagnets close to the normal metal may lead to magnetic flux lines penetrating inside the normal metal, which can orient the magnetic impurities in a preferential direction. We implicitly assume that the temperature is high enough so that the impurities have no preferential orientation. We consider a one dimensional model because only in this geometry can we decouple the spin and charge sectors of the Boltzmann equation. We assume that the interfaces between the ferromagnets and the normal metal are sharp, and that the exchange field has a step function variation at the interface. We note $f_{R, L}^{\sigma}(E, x)$ the semi classical distribution function of right/left moving spin- $\sigma$ electrons with an energy $E$ at position $x$.

The Boltzmann equation in the relaxation time approximation reads

$$
\frac{\partial}{\partial x}\left(\begin{array}{c}
f_{R}^{\uparrow}(E, x) \\
f_{L}^{\uparrow}(E, x) \\
f_{R}^{\downarrow}(E, x) \\
f_{L}^{\downarrow}(E, x)
\end{array}\right)=\left(\begin{array}{cccc}
-\left(r+r_{s}+r_{s}^{\prime}\right) & r & r_{s} & r_{s}^{\prime} \\
-r & r+r_{s}+r_{s}^{\prime} & -r_{s}^{\prime} & -r_{s} \\
r_{s} & r_{s}^{\prime} & -\left(r+r_{s}+r_{s}^{\prime}\right) & r \\
-r_{s}^{\prime} & -r_{s} & -r & r+r_{s}+r_{s}^{\prime}
\end{array}\right)\left(\begin{array}{c}
f_{R}^{\uparrow}(E, x) \\
f_{L}^{\uparrow}(E, x) \\
f_{R}^{\downarrow}(E, x) \\
f_{L}^{\downarrow}(E, x)
\end{array}\right),
$$

where we have discarded the term involving the electric field. This is valid if the temperature of the electrodes is larger than the applied voltage, in which case the electronic gas has a temperature identical to the one of the electrodes [15, 16]. Therefore, we should consider a finite temperature and calculate the low voltage conductance in the regime $e V \ll T$. In practise, we consider the limit $T \rightarrow 0$, and calculate the linear conductance. The coefficients $r, r_{s}$ and $r_{s}^{\prime}$ in Eq. 1 denote respectively the rate of backscattering without spin-flip, the rate of forward scattering with spin-flip, and the rate of backward scattering with spin-flip. The coefficients can be related to the $q=0$ and $q=2 k_{f}$ components of the microscopic scattering potential (see the Appendix). 
We now explicit the boundary conditions. For this purpose, let us consider an interface between a ferromagnet in the region $x<0$ and a normal metal in the region $x>0$, and include interface scattering under the form of repulsive potential $H \delta(x)$ [17]. Let us first consider a spin-up electron incoming from the left ferromagnet, and denote by $b^{\uparrow}$ and $t^{\uparrow}$ the backscattering and transmission coefficients. The wave function in the region $x<0$ is $\psi_{L}(x)=\exp \left(i k^{\uparrow} x\right)+b^{\uparrow} \exp \left(-i k^{\uparrow} x\right)$, and the wave function in the region $x>0$ is $\psi_{R}(x)=t^{\uparrow} \exp (i k x)$. The matching equations are $\psi_{L}(0)=$ $\psi_{R}(0)=\psi(0)$ and $\partial \psi_{R}(0) / \partial x-\partial \psi_{L}(0) / \partial x=\left(2 m H / \hbar^{2}\right) \psi(0)$, from what we deduce

$$
t^{\uparrow}=\frac{2 i k^{\uparrow}}{i\left(k+k^{\uparrow}\right)-2 m H / \hbar^{2}}, \text { and } b^{\uparrow}=\frac{i\left(k^{\uparrow}-k\right)+2 m H / \hbar^{2}}{i\left(k+k^{\uparrow}\right)-2 m H / \hbar^{2}} \text {. }
$$

The probability current conservation can be verified easily: $k^{\uparrow}=k^{\uparrow}\left|b^{\uparrow}\right|^{2}+k\left|t^{\uparrow}\right|^{2}$. The spin-up conductance is found to be $G^{\uparrow}=\left(e^{2} / h\right)\left|T^{\uparrow}\right|^{2}$, with the transmission coefficient

$$
T^{\uparrow}=\frac{k}{k^{\uparrow}}\left|t^{\uparrow}\right|^{2}=\frac{4 k k^{\uparrow}}{\left(k+k^{\uparrow}\right)^{2}+\left[\frac{2 m H}{\hbar^{2}}\right]^{2}} .
$$

The backscattering coefficient is $B^{\uparrow}=1-T^{\uparrow}$. In the spin-down sector, we obtain $T^{\downarrow}$ and $B^{\downarrow}$ by substituting $k^{\uparrow}$ with $k^{\downarrow}$ in Eq. 2. This provides the boundary conditions for the Boltzmann equation:

$$
\begin{aligned}
f_{R}^{\uparrow}(E, 0) & =T^{\downarrow} f_{T}(E-e V)+\left(1-T^{\downarrow}\right) f_{L}^{\uparrow}(E, 0) \\
f_{R}^{\downarrow}(E, 0) & =T^{\uparrow} f_{T}(E-e V)+\left(1-T^{\uparrow}\right) f_{L}^{\downarrow}(E, 0) \\
f_{L}^{\uparrow}(E, L) & =T^{\uparrow} f_{T}(E)+\left(1-T^{\uparrow}\right) f_{R}^{\uparrow}(E, L) \\
f_{L}^{\downarrow}(E, L) & =T^{\downarrow} f_{T}(E)+\left(1-T^{\downarrow}\right) f_{R}^{\downarrow}(E, L),
\end{aligned}
$$

where the left and right ferromagnets are assumed to be in equilibrium and $f_{T}(E)$ denotes the FermiDirac distribution function. We consider $T^{\uparrow}$ and $T^{\downarrow}$ to be independent of energy, which amounts to considering the wave vectors $k$ and $k^{\uparrow}$ in Eq. 2 to be on the Fermi surface. Eqs. 3 - 6 provide a simple form for the spin-up and spin-down currents at positions $x=0, L$. For instance at $x=L$, we have

$$
\begin{aligned}
I^{\uparrow}(L) & =T^{\uparrow} \frac{e}{h} \int\left[f_{R}^{\uparrow}(E, L)-f_{T}(E)\right] d E \\
I^{\downarrow}(L) & =T^{\downarrow} \frac{e}{h} \int\left[f_{R}^{\downarrow}(E, L)-f_{T}(E)\right] d E .
\end{aligned}
$$

The Boltzmann equation Eq. 10 and the boundary conditions Eqs. 3- 6 lead to eight equations for eight variables $f_{R, L}^{\uparrow, \downarrow}(E, x=0, L)$. We now solve these equations directly and discuss their physics.

\subsection{Solution of the Boltzmann equation}

The $4 \times 4$ Boltzmann equation can block diagonalized into $2 \times 2$ blocks by changing variables to the charge and spin combinations $X_{R, L}=f_{R, L}^{\uparrow}+f_{R, L}^{\downarrow}$ and $Y_{R, L}=f_{R, L}^{\uparrow}-f_{R, L}^{\downarrow}$. This spin-charge 
decoupling allows to solve exactly the Boltzmann equation. We find

$$
\frac{\partial}{\partial x}\left(\begin{array}{c}
X_{R} \\
X_{L}
\end{array}\right)=\frac{1}{l}\left(\begin{array}{cc}
-1 & 1 \\
-1 & 1
\end{array}\right)\left(\begin{array}{c}
X_{R} \\
X_{L}
\end{array}\right) \text {, and } \frac{\partial}{\partial x}\left(\begin{array}{c}
Y_{R} \\
Y_{L}
\end{array}\right)=\left(\begin{array}{cc}
-a & b \\
-b & a
\end{array}\right)\left(\begin{array}{c}
Y_{R} \\
Y_{L}
\end{array}\right),
$$

with $l=1 /\left(r+r_{s}^{\prime}\right)$ the mean free path, and $a=r+2 r_{s}+r_{s}^{\prime}, b=r-r_{s}^{\prime}$. The $2 \times 2$ block equations can be easily integrated to obtain

$$
\left(\begin{array}{c}
X_{R}(L) \\
X_{L}(L)
\end{array}\right)=\left(\begin{array}{cc}
1-x & x \\
-x & 1+x
\end{array}\right)\left(\begin{array}{c}
X_{R}(0) \\
X_{L}(0)
\end{array}\right)
$$

with $x=L / l$. Similarly,

$$
\left(\begin{array}{c}
Y_{R}(L) \\
Y_{L}(L)
\end{array}\right)=\hat{T}\left(\begin{array}{c}
X_{R}(0) \\
X_{L}(0)
\end{array}\right) \text {, with } \hat{T}=\left(\begin{array}{cc}
t & u \\
-u & \bar{t}
\end{array}\right)
$$

where $t=\cosh (\lambda L)-\alpha \sinh (\lambda L), \bar{t}=\cosh (\lambda L)+\alpha \sinh (\lambda L)$, and $u=\beta \sinh (\lambda L)$. We used the notation $\alpha=a / \lambda, \beta=b / \lambda$, and $\lambda=\sqrt{a^{2}-b^{2}}$. Next, we combine the boundary conditions Eqs. $3-6$ to Eq. 10 to obtain an expression for $f_{R}^{\uparrow}(E, 0)-f_{R}^{\downarrow}(E, 0)$ and $f_{L}^{\uparrow}(E, 0)-f_{L}^{\downarrow}(E, 0)$ as a function of $f_{R}^{\uparrow}(E, L)$ and $f_{R}^{\downarrow}(E, L)$. Once injected into Eq. 11, these relations lead to

$$
\begin{aligned}
\hat{M}\left(\begin{array}{c}
f_{R}^{\uparrow}(E, L) \\
f_{R}^{\downarrow}(E, L)
\end{array}\right) & =2 f_{T}(E-e V) \hat{T}\left(\begin{array}{c}
T^{\uparrow}+T^{\downarrow}-2 T^{\uparrow} T^{\downarrow} \\
T^{\uparrow}+T^{\downarrow}
\end{array}\right) \\
& -\quad f_{T}(E)\left\{\left(T^{\uparrow}+T^{\downarrow}\right) \hat{T}\left(\begin{array}{c}
2\left(T^{\uparrow}+T^{\downarrow}-T^{\uparrow} T^{\downarrow}-1\right)-x\left(T^{\uparrow}+T^{\downarrow}-2 T^{\uparrow} T^{\downarrow}\right) \\
-2+T^{\uparrow}+T^{\downarrow}-x\left(T^{\uparrow}+T^{\downarrow}\right)
\end{array}\right)\right. \\
& \left.-\left(T^{\uparrow}-T^{\downarrow}\right)^{2}\left(\begin{array}{c}
0 \\
1
\end{array}\right)\right\} .
\end{aligned}
$$

The matrix $\hat{M}$ appearing in the left hand side of Eq. 12 is

$$
\hat{M}=\hat{T}\left(\begin{array}{cc}
A^{\uparrow} & A^{\downarrow} \\
B^{\uparrow} & B^{\downarrow}
\end{array}\right)+\left(T^{\uparrow}-T^{\downarrow}\right)\left(\begin{array}{cc}
-1 & 1 \\
-1+T^{\uparrow} & 1-T^{\downarrow}
\end{array}\right),
$$

with the coefficients

$$
\begin{aligned}
& A^{\uparrow}=3 T^{\uparrow}-4 T^{\uparrow} T^{\downarrow}+T^{\downarrow}-2\left(T^{\uparrow}\right)^{2}+2\left(T^{\uparrow}\right)^{2} T^{\downarrow}+x T^{\uparrow}\left[T^{\uparrow}-2 T^{\uparrow} T^{\downarrow}+T^{\downarrow}\right] \\
& B^{\uparrow}=3 T^{\uparrow}+T^{\downarrow}-\left(T^{\uparrow}\right)^{2}-T^{\uparrow} T^{\downarrow}+x\left[\left(T^{\uparrow}\right)^{2}+T^{\uparrow} T^{\downarrow}\right] .
\end{aligned}
$$

The expression of $A^{\downarrow}$ is obtained by exchanging $T^{\uparrow}$ and $T^{\downarrow}$ in Eq. 14. Similarly, $B^{\downarrow}$ is obtained from $B^{\uparrow}$ by exchanging $T^{\uparrow}$ and $T^{\downarrow}$ in Eq. 15 .

\subsection{Fully polarized limit}

We first consider the solution Eq. 12 in the case of fully polarized ferromagnets with high transparency contacts: $H=0, k^{\downarrow}=T^{\downarrow}=0, k^{\uparrow}=k$, leading to $T^{\uparrow}=1$. In this limit, only a spin-up current 


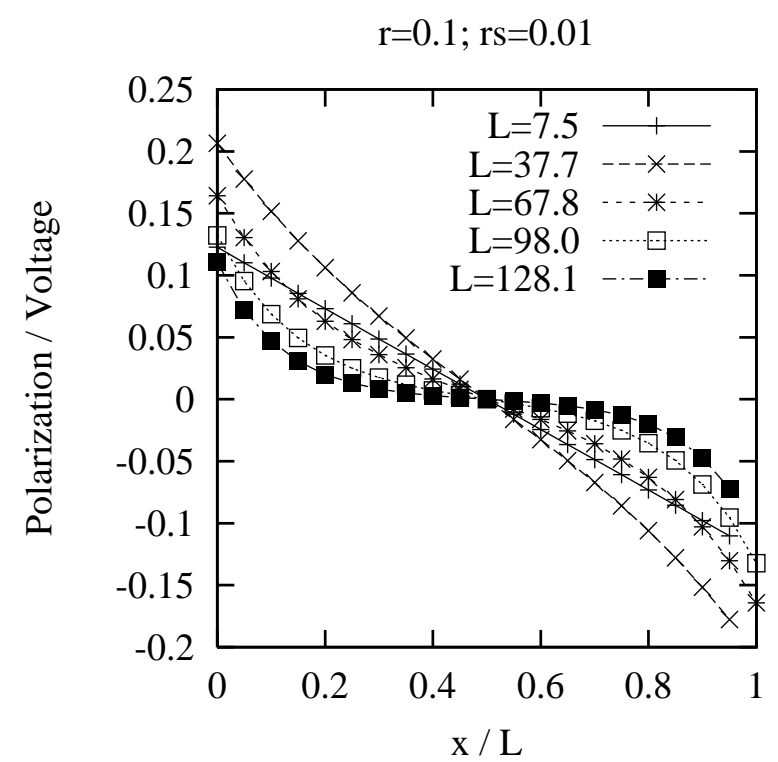

Figure 2: Spin polarization profile inside the diffusive conductor, with $r=0.1, r_{s}=0.01$, and increasing values of $L$. The spin polarization is normalized to the applied voltage, and the coordinate along the wire is normalized to the total length. We have $1 / \lambda=15.8$.

can enter the ferromagnet at $x=L$. This is expected on physical grounds, and it can be verified explicitly on the form Eq. 8 of the spin-down current. The total current is found to be

$$
I=\frac{e}{h} \int d E \frac{2\left[f_{T}(E-e V)-f_{T}(E)\right] \sinh \left[2 \sqrt{r_{s}\left(r_{s}+r\right)} L\right]}{(2+r L) \sinh \left[2 \sqrt{r_{s}\left(r_{s}+r\right)} L\right]+\sqrt{\frac{r}{r_{s}}+1}\left\{\cosh \left[2 \sqrt{r_{s}\left(r_{s}+r\right)} L\right]+1\right\}},
$$

where we considered only the forward scattering spin flip processes $\left(r_{s}^{\prime}=0\right)$, and assumed that $r_{s} \ll r$, in which case the elastic mean free path $l=1 / r$ is much below the spin-flip length $l_{\mathrm{sf}}=$ $1 /\left[2 \sqrt{r_{s}\left(r_{s}+r\right)}\right]$. If $L$ is small compared to $l_{\mathrm{sf}}$, the conductance $G \sim 2 \frac{e^{2}}{h} r_{s} L$ shows an inverse Drude behavior.

\subsection{Spin polarization profile}

The spin polarization profile in the diffusive wire can be calculated in a straightforward fashion from the solution of the Boltzmann equation. Once we know the distribution functions at one extremity of the wire, we can use Eqs. 9 to propagate the solution to an arbitrary point. The resulting spin polarization inside the wire is proportional to the applied voltage, and is shown on Fig. 目 for various values of $L$. When $L>l_{\mathrm{sf}}$, there is a plateau in the spin polarization in the middle of the wire. In the opposite inverse Drude regime, there is no such plateau. 


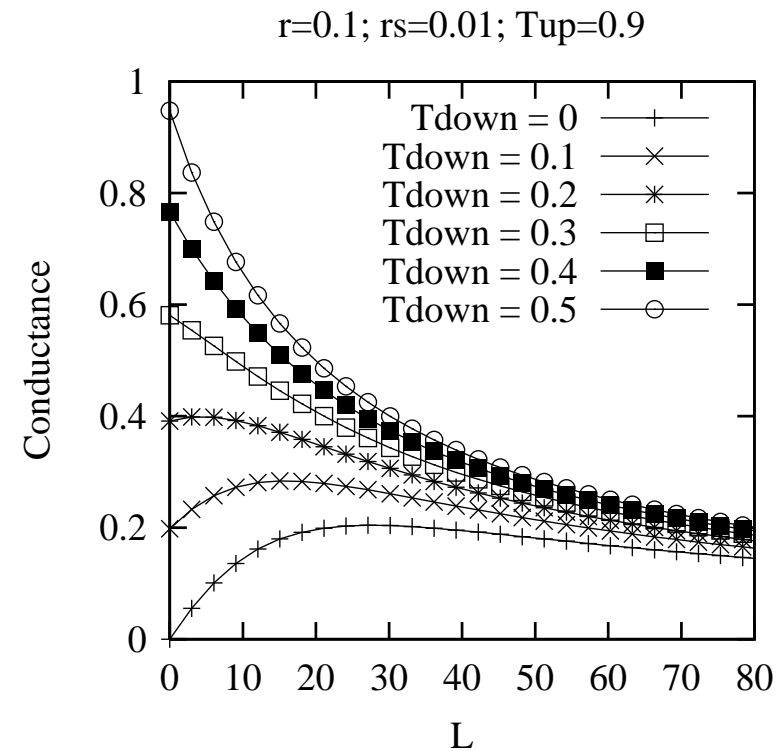

Figure 3: Variations of the conductance as a function of the length $L$ of the diffusive wire, with decreasing spin polarizations $T^{\downarrow}=0,0.1,0.2,0.3,0.4,0.5$, and the parameters $T^{\uparrow}=0.9, r=0.1$ and $r_{s}=0.01$. With a strong spin polarization, the conductance increases with $L$ below $L_{c}$. With a weak spin polarization, the conductance decreases monotonically with $L\left(L_{c}=0\right)$.

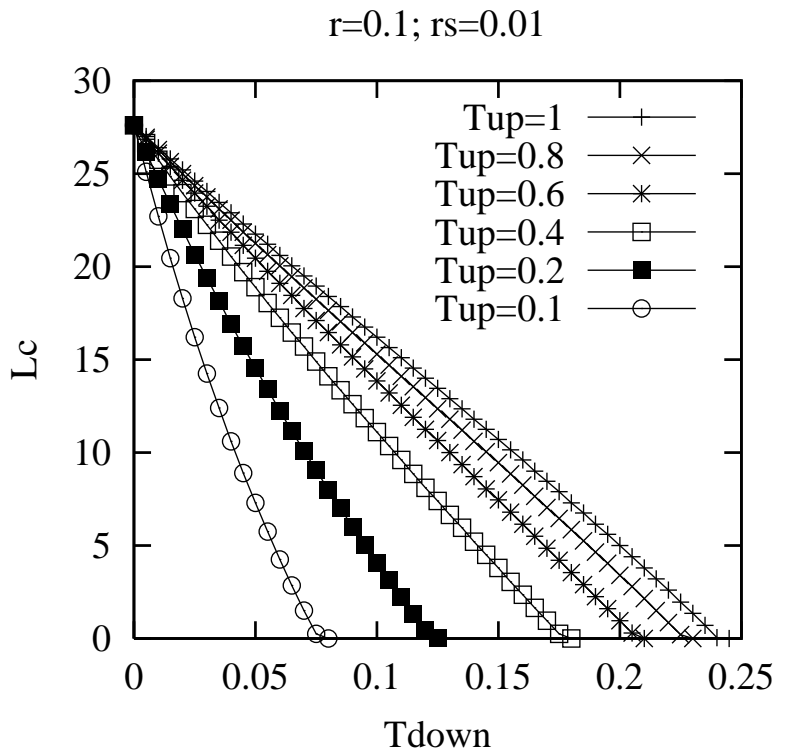

Figure 4: Variations of $L_{c}$ as a function of $T^{\downarrow}$, with increasing spin polarizations $T^{\uparrow}=0.1,0.2,0.4$, $0.6,0.8,1$, and the parameters $r=0.1$ and $r_{s}=0.01$. With a given $T^{\uparrow}$, the curve $L_{c}\left(T^{\downarrow}\right)$ separates two regions: (i) small- $L$, small- $T^{\downarrow}$ : the conductance increases with $L$; and (ii) large- $L$, large- $T^{\downarrow}$ : the conductance decreases with $L$. 


\subsection{Effect of a partial spin polarization}

Now, we consider the effect of a partial spin polarization in the ferromagnet. It is expected on physical grounds that a decreasing spin polarization tends to suppress the inverse Drude scaling because this regime is clearly absent in the spin unpolarized case. This is visible on Fig. 3 where we plotted the conductance as a function of the length of the diffusive wire for decreasing spin polarizations. With an arbitrary polarization, there exists a critical length scale $L_{c}$ such that the conductance increases with $L$ below $L_{c}$, and decreases with $L$ above $L_{c}$. There exists also a critical value of $T^{\downarrow}$ such that

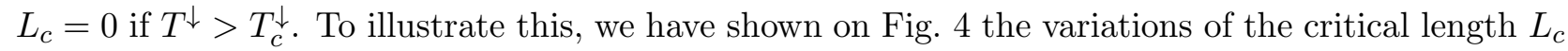
as a function of the parameter $T^{\downarrow}$. When $T^{\downarrow}$ increases, $L_{c}$ decreases: the maximum in $G(L)$ occurs for a smaller $L_{c}$. When $T^{\downarrow}$ is above a critical value $T_{c}^{\downarrow}$, the conductance decreases monotonically with $L$.

We now describe the effect of a partial spin polarization on the basis of a small- $T^{\downarrow}$ expansion. The strategy is to express the current to order $L$ and determine whether the conductance increases or decreases with $L$. We expand the current to first order in the two parameters $K=\lambda L$ and $x=L / l$, and retain the coefficients of this expansion to leading order in $T^{\downarrow}$. It is first instructive to carry out the expansion with $L=0$, and therefore $K=x=0$. It is visible on Eqs. 7 , 8 that a prefactor $T^{\uparrow}$ enters the spin-up current, and a prefactor $T^{\downarrow}$ enters the spin-down current. We should then express $f_{R}^{\uparrow}(E, L)$ to first order in $T^{\downarrow}$ while $f_{R}^{\downarrow}(E, L)$ should be expressed to order $\left(T^{\downarrow}\right)^{0}$. The spin-up and spin-down channels appear to play an asymmetric role. Nevertheless, the final expression of the conductance is identical in the spin-up and spin-down channels. An intermediate step in the calculation of $f_{R}^{\uparrow}(E, L)$ is the derivation of $\operatorname{Det} \hat{M}$ to order $T^{\downarrow}$ (see Eq. 13):

$$
\operatorname{Det} \hat{M}=-4\left(T^{\uparrow}\right)^{3}\left\{1-T^{\downarrow}\left(\frac{2 T^{\uparrow}-1}{T^{\uparrow}}\right)\right\},
$$

leading to an identical current in both spin channels:

$$
I^{\uparrow}=I^{\downarrow}=T^{\downarrow} \frac{e}{h} \int\left[f_{T}(E-e V)-f_{T}(E)\right] d E .
$$

Now we consider a diffusive wire with a finite length $L$, and expand the current to first order in $x$ and $K$, and to leading order in $T^{\downarrow}$. The determinant of the matrix $\hat{M}$ in Eq. 12 is found to be

$$
\operatorname{Det} \hat{M}=-4\left(T^{\uparrow}\right)^{3}\left\{1-T^{\downarrow}\left(\frac{2 T^{\uparrow}-1}{T^{\uparrow}}\right)\right\}-4\left(T^{\uparrow}\right)^{2}(\alpha-\beta)\left(2-T^{\uparrow}\right) K-4 x\left(T^{\uparrow}\right)^{2} T^{\downarrow} .
$$

Next we expand the spin-up current to order $L$ to obtain

$$
I^{\uparrow}=\frac{-4\left(T^{\uparrow}\right)^{3}}{\operatorname{Det} \hat{M}}\left(T^{\downarrow}-(\alpha-\beta) K\right) \simeq T^{\downarrow}\left[1+K \frac{\alpha-\beta}{T^{\downarrow}}-x \frac{T^{\downarrow}}{T^{\uparrow}}\right] .
$$

If $T^{\downarrow}$ is small, the current increases with $L$ while it decreases with $L$ if $T^{\downarrow}$ is large. The transition between these two behaviors is obtained for $T_{c}^{\downarrow}=\sqrt{\left(2 r_{s} / r\right) T^{\uparrow}}$, compatible with the behavior shown on Fig. 4 . 


\subsection{Replacement of one of the ferromagnets by a normal metal}

We now consider the situation where we replace the left-hand-side ferromagnet on Fig. 1 1 by a normal metal. In the presence of high transparency contacts, the conductance of this junction is of order $e^{2} / h$ in the absence of diffusion while it is of order $\left(e^{2} / h\right) T^{\downarrow}$ in the spin valve geometry on Fig. 1. Replacing one of the ferromagnets by a normal metal is expected to suppress the inverse Drude scaling. The boundary conditions appropriate to describe this situation are

$$
\begin{aligned}
f_{R}^{\uparrow}(E, 0) & =T f_{T}(E-e V)+(1-T) f_{L}^{\uparrow}(E, 0) \\
f_{R}^{\downarrow}(E, 0) & =T f_{T}(E-e V)+(1-T) f_{L}^{\downarrow}(E, 0) \\
f_{L}^{\uparrow}(E, L) & =T^{\uparrow} f_{T}(E)+\left(1-T^{\uparrow}\right) f_{R}^{\uparrow}(E, 0) \\
f_{L}^{\downarrow}(E, L) & =T^{\downarrow} f_{T}(E)+\left(1-T^{\downarrow}\right) f_{R}^{\downarrow}(E, 0),
\end{aligned}
$$

that should be solved together with Eqs. 9. The solution is found to be

$$
\hat{N}\left(\begin{array}{c}
f_{R}^{\uparrow}(E, L) \\
f_{R}^{\downarrow}(E, L)
\end{array}\right)=\left(\begin{array}{c}
\left(T^{\uparrow}+T^{\downarrow}\right)(1-T+x T) \\
\left(T^{\uparrow}-T^{\downarrow}\right)(u+t(1-T))
\end{array}\right) f_{T}(E)+2 T\left(\begin{array}{c}
1 \\
0
\end{array}\right) f_{T}(E-e V),
$$

with

$$
\hat{N}=\left(\begin{array}{cc}
C^{\uparrow} & C^{\downarrow} \\
D^{\uparrow} & -D^{\downarrow}
\end{array}\right)
$$

and

$$
\begin{aligned}
C^{\uparrow} & =T+T^{\uparrow}-T T^{\uparrow}+x T T^{\uparrow} \\
D^{\uparrow} & =\bar{t}-u(1-T)-\left(1-T^{\uparrow}\right)(t(1-T)+u) .
\end{aligned}
$$

We have plotted on Fig. 5 the conductance of the junction with high transparency contacts, where it is visible that the conductance decreases monotonically with the length of the diffusive wire.

Now, reducing the contact transparency restores a regime in which the conductance increases with the size of the diffusive wire. This is visible on Fig. 6 where we used $T=0.01$ and $T^{\uparrow}=1$. Again, the inverse Drude scaling is obtained for the smallest values of $T^{\downarrow}$ (with strongly polarized magnets).

\section{Quantum coherent transport: a single magnetic impurity "spin- flip Fabry Perot interferometer"}

\subsection{Matching equations}

We now consider a single magnetic impurity at $x=0$ in a normal metal, in the presence of a normal metal - ferromagnet interface at $x=a$ (see Fig. 7). The purpose of this calculation is to study a model in which the interplay between multiple reflections and phase coherence is treated exactly, and 


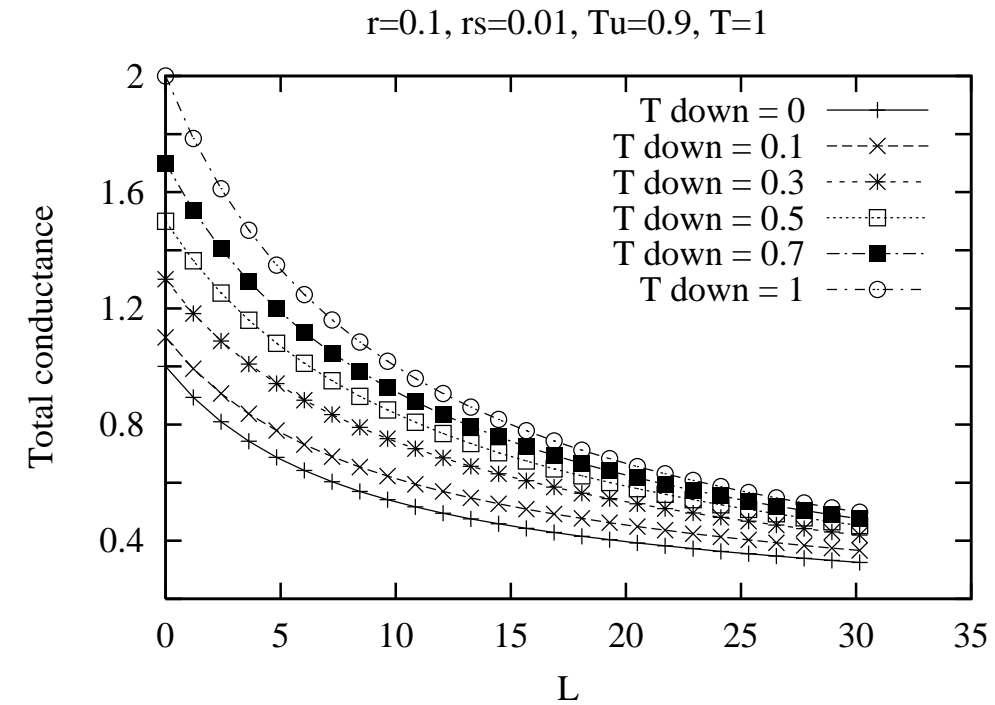

Figure 5: Conductance of the junction with a diffusive wire connected to a normal metal and a ferromagnet, with high transparency contacts: $T=T^{\uparrow}=1$. It is visible that the conductance decreases monotonically with the length of the diffusive wire.

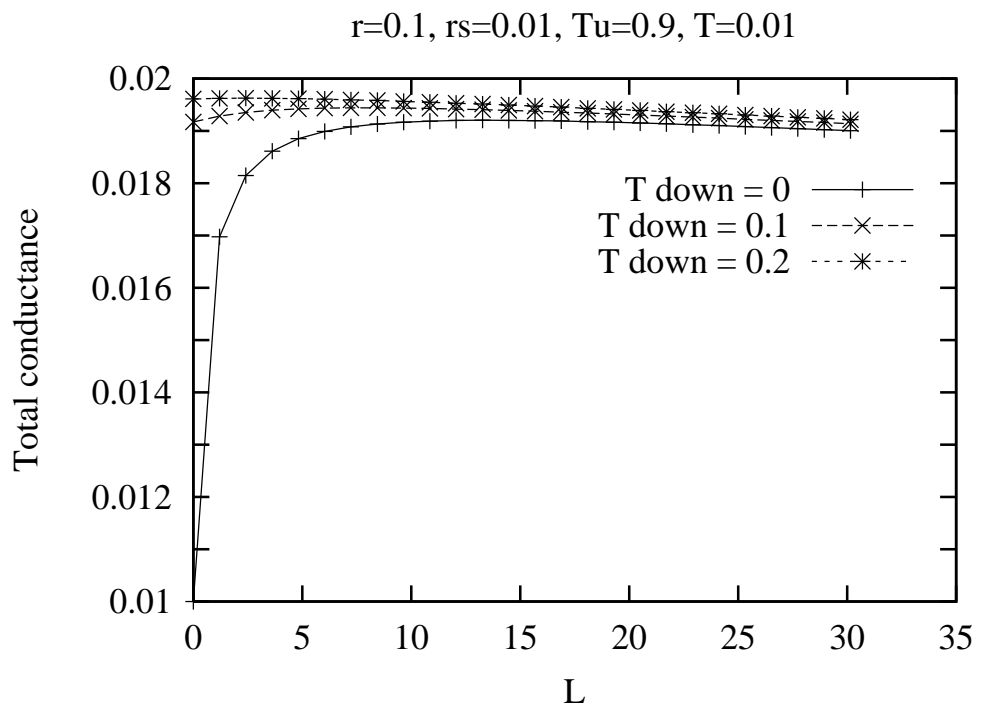

Figure 6: Conductance of the junction with a diffusive wire connected to a normal metal and a ferromagnet, with low transparency contacts: $T=0.01$, and $T^{\uparrow}=1$. A regime with a conductance increasing with the length of the diffusive wire is visible for small values of the parameter $T^{\downarrow}$. 


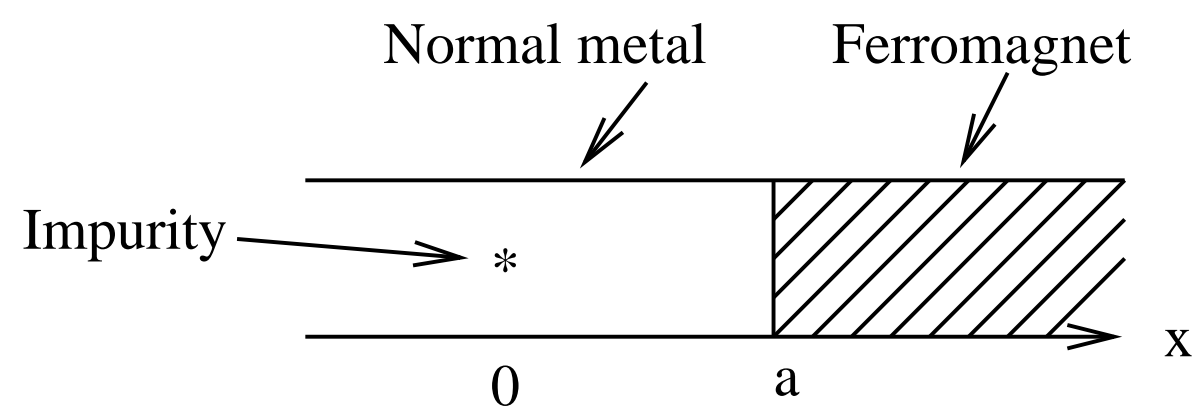

Figure 7: The system considered in section 3. The impurity is in the normal metal at a distance $a$ away from the ferromagnet. We have represented a quasi one dimensional geometry while the calculation is made in a one dimensional geometry.

to determine whether there exists a signature of spin accumulation in the quantum coherent regime. We find that the quantum model behaves like a Fabry Perot interferometer, with a resonance in the spin flip channels. This can be viewed as the signature of spin accumulation in the quantum coherent regime. We neglect the Kondo effect because we want to describe a situation in which the temperature is above the Kondo temperature. The conduction electrons are scattered through the Hamiltonian $\mathcal{H}=V_{0}+V_{1} \mathbf{S}_{i} . \mathbf{s}$, where $\mathbf{S}_{i}$ is the impurity spin and $\mathbf{s}$ the spin of the conduction electron, and we further assume a single channel geometry. This type of model has been used by Zhu and Wang [13] to investigate the effect of a magnetic impurity close to a normal metal - superconductor interface. The spin-up and spin-down wave functions are grouped in a two-component spinor $\hat{\psi}(x)$. Clearly, the impurity couples the spin-up and spin-down wave functions. The matching of the wave function at the impurity site reads

$$
\hat{\psi}\left(0^{+}\right)=\hat{\psi}\left(0^{-}\right) \text {, and } \frac{\partial \hat{\psi}}{\partial x}\left(0^{+}\right)-\frac{\partial \hat{\psi}}{\partial x}\left(0^{-}\right)=\frac{2 m}{\hbar^{2}}\left[\lambda \hat{1}+\mu \hat{\sigma}_{x}\right] \hat{\psi}(0)
$$

with $\lambda=V_{0}-V_{1} / 4$ and $\mu=V_{1} / 2$. The matching of the wave function at the ferromagnet boundary reads

$$
\hat{\psi}\left(a^{+}\right)=\hat{\psi}\left(a^{-}\right), \text {and } \frac{\partial \hat{\psi}}{\partial x}\left(a^{+}\right)-\frac{\partial \hat{\psi}}{\partial x}\left(a^{-}\right)=\frac{2 m}{\hbar^{2}} H \hat{\psi}(a),
$$

where we included a repulsive interface potential $H \delta(x-a)$ at the normal metal - ferromagnet interface. Eqs. 23, 24 generate eight constraints, for a set of eight transmission coefficients.

This calculation amounts to a resummation to all orders of a series of diagrams in which a conduction electron scatters onto the impurity, scatters back onto the interface, scatters again onto the impurity, ... (see Fig. 8 (a)). Note that the diagram with a hole in the intermediate state shown on Fig. 8 (b) generates another series which is not included in the calculation. If one wanted to describe the Kondo effect close to a ferromagnet interface, it would be crucial to incorporate the diagram on Fig. 8 (b), as well as inserting the interface scattering in this diagram. 
(a)

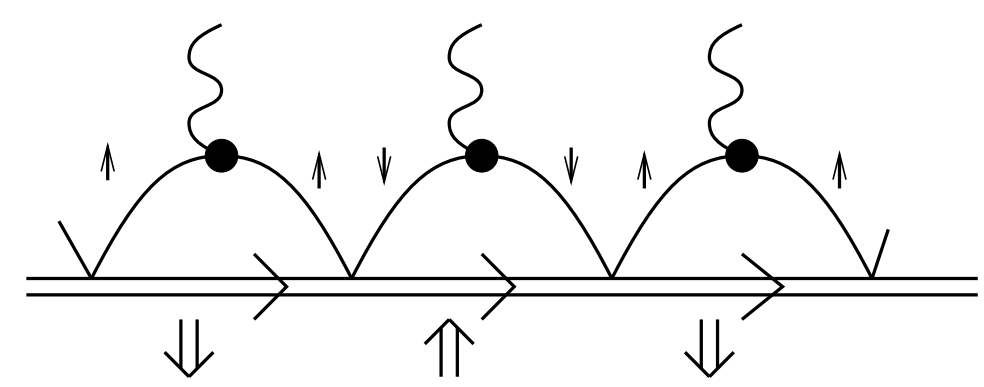

(b)

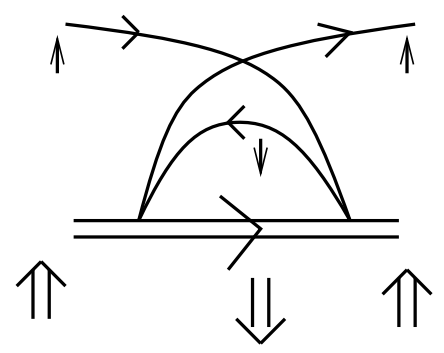

Figure 8: (a) The processes included in the Landauer calculation. The wavy lines indicate the scattering at the interface; and (b) A process with a hole in the intermediate state, not included in the calculation.

\subsection{Scattering in the total spin $S^{z}=0$ sectors}

\subsubsection{Incoming electron with a spin-up}

We first consider a spin-up electron incoming on the interface while the impurity is supposed to have initially a spin down. The wave functions are

$$
\begin{aligned}
& \hat{\psi}_{i \downarrow}^{e \uparrow}(x)=\left(\begin{array}{c}
1 \\
0
\end{array}\right) e^{i k x}+\left(\begin{array}{c}
b_{i \downarrow}^{e \uparrow \rightarrow e \uparrow} \\
b_{i \downarrow}^{e \uparrow \rightarrow} \rightarrow
\end{array}\right) e^{-i k x} \text { if } x<0 . \\
& \hat{\psi}_{i \downarrow}^{e \uparrow}(x)=\left(\begin{array}{c}
\alpha \\
\alpha^{\prime}
\end{array}\right) e^{i k x}+\left(\begin{array}{c}
\beta \\
\beta^{\prime}
\end{array}\right) e^{-i k x} \text { if } 0<x<a . \\
& \hat{\psi}_{i \downarrow}^{e \uparrow}(x)=t_{i \downarrow}^{e \uparrow \rightarrow e \uparrow}\left(\begin{array}{c}
1 \\
0
\end{array}\right) e^{i k^{\uparrow} x}+t_{i \downarrow}^{e \uparrow \rightarrow e \downarrow}\left(\begin{array}{c}
0 \\
1
\end{array}\right) e^{i k^{\downarrow} x} \text { if } x>a,
\end{aligned}
$$

where $k^{\uparrow}$ and $k^{\downarrow}$ denote the spin-up and spin-down Fermi wave vectors in the ferromagnet. In the notation of the transmission coefficients, the superscript denotes the initial and final spin orientations of the conduction electron while the subscript denotes the initial orientation of the impurity. The solution of the matching equations is straightforward, and we find the transmission coefficients

$$
\begin{aligned}
t_{i \downarrow}^{e \uparrow \rightarrow e \uparrow} & =\frac{1}{\mathcal{D} A^{\uparrow}}\left[\bar{A}(1+i z) X^{\downarrow}+A i z Y^{\downarrow}\right] \\
t_{i \downarrow}^{e \uparrow \rightarrow e \downarrow} & =-\frac{1}{\mathcal{D} A^{\downarrow}} i z^{\prime}\left[\bar{A} X^{\uparrow}+A Y^{\uparrow}\right] .
\end{aligned}
$$


We used the notation $X^{\sigma}=\frac{1}{2}+i Z+\frac{Z}{2 Z^{\sigma}}, Y^{\sigma}=\frac{1}{2}-\left(i Z+\frac{Z}{2 Z^{\sigma}}\right), A=\exp (i k a), A^{\sigma}=\exp \left(i k^{\sigma} a\right)$. The dimensionless scattering potentials in Eqs. 28, 29 are $z=m \lambda /\left(\hbar^{2} k\right)$ and $z^{\prime}=m \mu /\left(\hbar^{2} k\right)$ at the impurity site, and $Z=m H /\left(\hbar^{2} k\right), Z^{\sigma}=m H /\left(\hbar^{2} k^{\sigma}\right)$ at the normal metal - ferromagnet interface. The denominator $\mathcal{D}$ in Eqs. 28, 29 is

$$
\mathcal{D}=X^{\uparrow} X^{\downarrow}(\bar{A})^{2}\left[1-z^{2}+\left(z^{\prime}\right)^{2}+2 i z\right]+\left(X^{\uparrow} Y^{\downarrow}+X^{\downarrow} Y^{\uparrow}\right)\left[-z^{2}+\left(z^{\prime}\right)^{2}+i z\right]+Y^{\uparrow} Y^{\downarrow} A^{2}\left[-z^{2}+\left(z^{\prime}\right)^{2}\right] .
$$

\subsubsection{Incoming electron with a spin-down}

We consider now an incoming electron with a spin-down while the impurity has initially a spin-up. The wave functions are

$$
\begin{aligned}
& \hat{\psi}_{i \uparrow}^{e \downarrow}(x)=\left(\begin{array}{c}
0 \\
1
\end{array}\right) e^{i k x}+\left(\begin{array}{c}
b_{i \uparrow}^{e \downarrow \rightarrow e \uparrow} \\
b_{i \uparrow}^{e \downarrow \rightarrow e \downarrow}
\end{array}\right) e^{-i k x} \text { if } x<0 . \\
& \hat{\psi}_{i \uparrow}^{e \downarrow}(x)=\left(\begin{array}{c}
\alpha^{\prime} \\
\alpha
\end{array}\right) e^{i k x}+\left(\begin{array}{c}
\beta^{\prime} \\
\beta
\end{array}\right) e^{-i k x} \text { if } 0<x<a . \\
& \hat{\psi}_{i \uparrow}^{e \downarrow}(x)=t_{i \uparrow}^{e \downarrow \rightarrow e \downarrow}\left(\begin{array}{c}
0 \\
1
\end{array}\right) e^{i k \downarrow x}+t_{i \uparrow}^{e \downarrow \rightarrow e \uparrow}\left(\begin{array}{c}
1 \\
0
\end{array}\right) e^{i k \uparrow} \text { if } x>a .
\end{aligned}
$$

The equations for $t_{i \uparrow}^{e \downarrow \rightarrow e \uparrow}$ and $t_{i \uparrow}^{e \downarrow \rightarrow e \downarrow}$ are obtained from the ones in section 3.2.1 under the transformation $A^{\uparrow} \leftrightarrow A^{\downarrow}$, and $Z^{\uparrow} \leftrightarrow Z^{\downarrow}$. The amplitude for transmission in the ferromagnet is

$$
\begin{aligned}
t_{i \uparrow}^{e \downarrow \rightarrow e \uparrow} & =-\frac{1}{\mathcal{D} A^{\uparrow}} i z^{\prime}\left[\bar{A} X^{\downarrow}+A Y^{\downarrow}\right] \\
t_{i \uparrow}^{e \downarrow \rightarrow e \downarrow} & =\frac{1}{\mathcal{D} A^{\downarrow}}\left[\bar{A}(1+i z) X^{\uparrow}+A i z Y^{\uparrow}\right] .
\end{aligned}
$$

\subsection{Scattering in the total spin $S^{z}= \pm 1$ sectors}

The incoming electron does not undergo spin-flip scattering in the sectors with a total spin $S^{z}= \pm 1$. The transmission coefficients in the sector $S^{z}=1$ is found to be

$$
t_{i \uparrow}^{e \uparrow \rightarrow e \uparrow}=\frac{1}{A^{\uparrow}\left[\bar{A}\left(1+i\left(z+z^{\prime}\right)\right) X^{\uparrow}+A i\left(z+z^{\prime}\right) Y^{\uparrow}\right]} .
$$

In the sector $S^{z}=-1$, we have

$$
t_{i \downarrow}^{e \downarrow \rightarrow e \downarrow}=\frac{1}{A^{\downarrow}\left[\bar{A}\left(1+i\left(z+z^{\prime}\right)\right) X^{\downarrow}+A i\left(z+z^{\prime}\right) Y^{\downarrow}\right]} .
$$

We can check easily that these forms of the transmission coefficients are identical to Eq. 28, with $z^{\prime}=0$, and the replacement $z \rightarrow z+z^{\prime}$. This is expected since there is no spin-dependent scattering in the limit $z^{\prime}=0$ of Eq. 28 . 


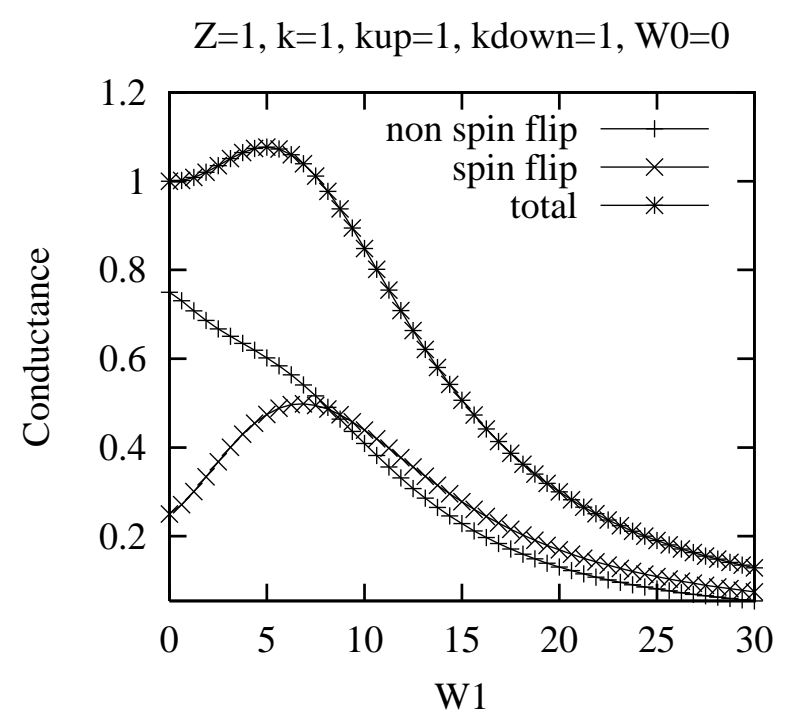

Figure 9: Spin-flip, non spin-flip, and total conductances (in units of $e^{2} / h$ ) of the quantum mechanical model of magnetic scattering close to a ferromagnet interface, with an unpolarized ferromagnet $\left(k=k^{\uparrow}=k^{\downarrow}=1\right)$ and the parameters $Z=1, a=100$ and $W_{0}=V_{0} / H=0$. The conductances are plotted as a function of $W_{1}=V_{1} / H$.

\subsection{Landauer formula}

We now evaluate the total conductance and assume that the incoming electron and impurity do not have any preferential direction. The conductance is the sum of four terms, weighted by the probability $\mathcal{P}=1 / 2$ to have a spin-up or spin-down impurity: $G=\left(G_{i \downarrow}^{e \uparrow}+G_{i \uparrow}^{e \downarrow}+G_{i \uparrow}^{e \uparrow}+G_{i \downarrow}^{e \downarrow}\right) / 2$, with

$$
\begin{aligned}
G_{i \downarrow}^{e \uparrow} & =\frac{e^{2}}{h}\left(\frac{k^{\uparrow}}{k}\left|t_{i \downarrow}^{e \uparrow \rightarrow e \uparrow}\right|^{2}+\frac{\operatorname{Re} k^{\downarrow}}{k}\left|t_{i \downarrow}^{e \uparrow \rightarrow e \downarrow}\right|^{2}\right) \\
G_{i \uparrow}^{e \downarrow} & =\frac{e^{2}}{h}\left(\frac{k^{\uparrow}}{k}\left|t_{i \uparrow}^{e \downarrow \rightarrow e \uparrow}\right|^{2}+\frac{\operatorname{Re} k^{\downarrow}}{k}\left|t_{i \uparrow}^{e \downarrow \rightarrow e \downarrow}\right|^{2}\right) \\
G_{i \uparrow}^{e \uparrow} & =\frac{e^{2}}{h} \frac{k^{\uparrow}}{k}\left|t_{i \uparrow}^{e \uparrow \rightarrow e \uparrow}\right|^{2} \\
G_{i \downarrow}^{e \downarrow} & =\frac{e^{2}}{h} \frac{\operatorname{Re} k^{\downarrow}}{k}\left|t_{i \downarrow}^{e \downarrow \rightarrow e \downarrow}\right|^{2} .
\end{aligned}
$$

We have incorporated the possibility of having a pure imaginary wave vector $k^{\downarrow}$, corresponding to an empty spin-down band.

\subsection{Resonances}

We consider the presence of a strong interface scattering at the metal - ferromagnet interface. The electron are multiply reflected before they enter the ferromagnet, and therefore the resonator has 


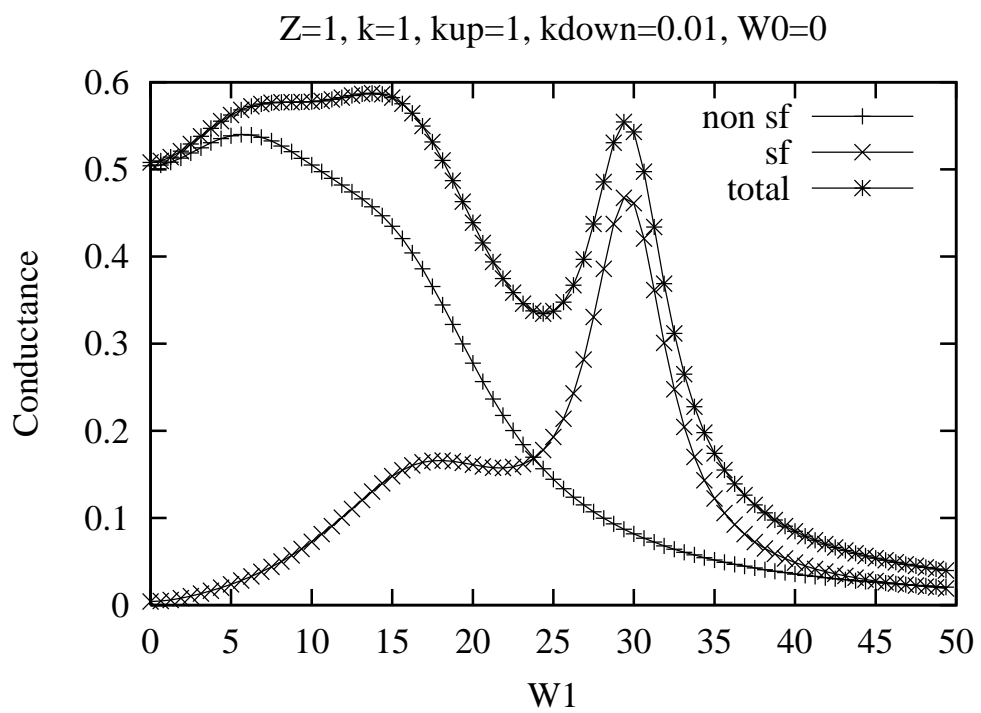

Figure 10: Spin-flip, non spin-flip, and total conductances (in units of $e^{2} / h$ ) of the quantum mechanical model of magnetic scattering close to a ferromagnet interface, with a strongly polarized ferromagnet $\left(k=k^{\uparrow}=1, k^{\downarrow}=0.01\right)$ and the parameters $Z=1, a=100$ and $W_{0}=V_{0} / H=0$. The conductances are plotted as a function of $W_{1}=V_{1} / H$. A resonance, not present on Fig. 9, develops in the conductance upon spin polarizing the ferromagnet.

a high quality factor. We first choose the parameter $a$ in such a way that spin-flip scattering is resonant. As it is visible on Figs. 9 and 10, the presence of a spin polarization in the ferromagnet generates a resonance in the conductance, not present in the unpolarized situation. We have shown the conductance with $V_{0}=0$ but a similar behavior has been obtained with a finite $V_{0}$. The values of $k a$ for which a resonance occurs can be worked out by calculating the transmission coefficients in the limit of a large $z, z^{\prime}$. In this limit, we find

$$
\begin{aligned}
t_{i \uparrow}^{e \downarrow \rightarrow e \uparrow} & =-\frac{i z^{\prime} \bar{A}^{\uparrow}}{\left(\left(z^{\prime}\right)^{2}-z^{2}\right)} \frac{1}{\bar{A} X^{\uparrow}+A Y^{\uparrow}} \\
t_{i \downarrow}^{e \uparrow \rightarrow e \uparrow} & =\frac{i z \bar{A} \uparrow}{\left(z^{\prime}\right)^{2}-z^{2}} \overline{\bar{A} X^{\uparrow}+A Y^{\uparrow}} .
\end{aligned}
$$

The resonances occur when $\tan (k a)=1 /(i-2 Z)$. For a large $Z$, the resonances are close to the real axis: $\tan (k a)=-1 /(2 Z)$, in agreement with Fig. 11. The reason why the resonance appear to be sharp as a function of $a$ when $Z$ is large is that, even without spin flip scattering, the quality factor of such a resonator is large when $Z$ is large.

The occurrence of a parameter range in which a peak occurs in the spin flip conductance is intriguing. The presence of a specific physics in the spin flip channels can be already understood from the large- $z, z^{\prime}$ behavior, Eqs. 40, 41. Typically, one has $\left|t_{i \uparrow}^{e \downarrow \rightarrow e \uparrow}\right|^{2} \sim\left(z^{\prime}\right)^{2} /\left(\left(z^{\prime}\right)^{2}-z^{2}\right)^{2}$ and $\left|t_{i \uparrow}^{e \uparrow \rightarrow e \uparrow}\right|^{2} \sim(z)^{2} /\left(\left(z^{\prime}\right)^{2}-z^{2}\right)^{2}$. In the presence of spin flip scattering, one has $z \neq z^{\prime}$ and therefore a 


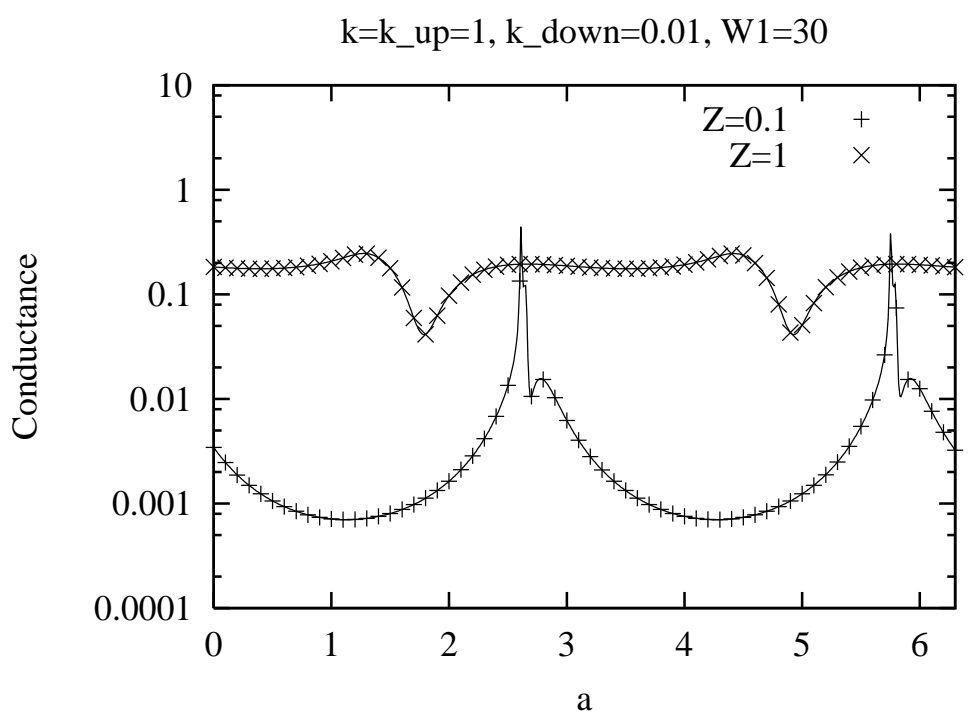

Figure 11: Spin flip conductance of the Fabry Perot model, as a function of $a$, with $k=k^{\uparrow}=1$, $k^{\downarrow}=0.01$. As it is visible, the conductance is a periodic function of $k a$ with a period $\pi$. The resonances with $Z=1$ are much sharper than with $Z=0.01$, as expected on physical grounds. The resonances occur when $\tan (k a) \simeq-1 /(2 Z)$.

different conductance in the spin flip and non spin flip channels.

\section{Conclusions}

To conclude, we have determined to what extend spin accumulation can result in an inverse Drude behavior in a semi classical spin valve model. Our treatment was based on an exact decoupling between the charge and spin sectors of the Boltzmann equation. We have addressed a similar question in a single impurity quantum model and found the existence of a resonance in the spin flip conductance. It is an open question to determine the quantum coherent behavior of a spin valve with a finite concentration of impurities.

\section{A Derivation of the Boltzmann equation with spin-flip scattering}

We give a brief derivation of the Boltzmann equation Eq. 1 in the presence of a spin-flip scattering potential. The derivation generalizes Ref. [18] to incorporate a spin-flip scattering self energy. The Dyson equation in the spin tensor Keldysh space reads $\left(\hat{G}_{0}^{-1}-\hat{\Sigma}\right)(1,2) \otimes \hat{G}(2)=\delta(1-2)$. The convolution includes a sum over coordinates, time, and spin. The kinetic equation is obtained from the difference of the Keldysh components of the Dyson equations and its conjugate:

$$
\left[\hat{G}_{0}^{-1}-\operatorname{Re} \hat{\Sigma}, \hat{G}^{K}\right]_{-}-\left[\hat{\Sigma}^{K}, \operatorname{Re} \hat{G}\right]_{-}=\frac{i}{2}\left[\hat{\Sigma}^{K}, \hat{A}\right]_{+}-\frac{i}{2}\left[\hat{\Gamma}, \hat{G}^{K}\right]_{+}
$$


(a)

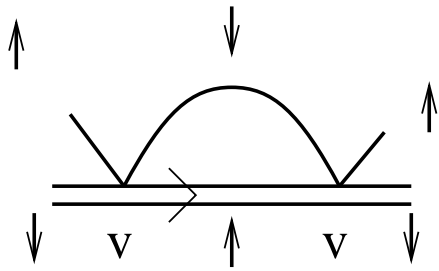

(b)

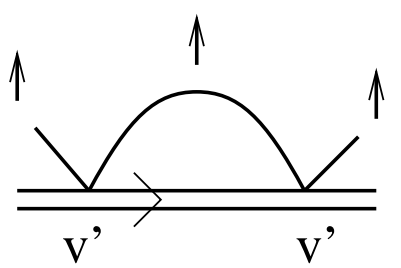

Figure 12: The self energy terms incorporated in the gradient expansion calculation. The term shown on Fig. 8 (b) generating the Kondo effect is not included.

with []$_{-}$and []$_{+}$denoting a commutator and an anticommutator respectively. We refer the reader to Ref. 18] for an explanation of the symbols used in Eq. 42. We use the the self energy shown on Fig. 12:

$$
\hat{\Sigma}_{\sigma}(\mathbf{p}, \mathbf{R}, T)=n_{i} \int \frac{d \mathbf{p}^{\prime}}{(2 \pi)^{3}}\left|v\left(\mathbf{p}-\mathbf{p}^{\prime}\right)\right|^{2} \hat{G}_{\sigma, \sigma}\left(\mathbf{p}^{\prime}, \mathbf{R}, T\right)+n_{i}^{\prime} \int \frac{d \mathbf{p}^{\prime}}{(2 \pi)^{3}}\left|v^{\prime}\left(\mathbf{p}-\mathbf{p}^{\prime}\right)\right|^{2} \hat{G}_{-\sigma,-\sigma}\left(\mathbf{p}^{\prime}, \mathbf{R}, T\right),
$$

with $n_{i}$ and $n_{i}^{\prime}$ the concentration of non magnetic and magnetic impurities. The first term in Eq. 43 describes non spin-flip scattering, and the second term describes spin-flip scattering. Notice that this self energy does not incorporate the Kondo effect since we do not incorporate the possibility of a having hole in the intermediate state.

We assume the self energy in Eq. 42 to be constant in space, and use the gradient expansion to first order

$$
(A \otimes B)_{\sigma, \sigma^{\prime}}(X, p) \simeq \sum_{\sigma_{2}}\left[1+\frac{i}{2}\left(\partial_{X}^{A} \partial_{p}^{B}-\partial_{p}^{A} \partial_{X}^{B}\right)\right] A_{\sigma, \sigma_{2}} B_{\sigma_{2}, \sigma^{\prime}}
$$

If $A$ and $B$ are symmetric in spin $A_{\sigma, \sigma^{\prime}}=A_{\sigma^{\prime}, \sigma}$, and $B_{\sigma, \sigma^{\prime}}=B_{\sigma^{\prime}, \sigma}$, the commutator reduces to the usual spinless Poisson bracket: $[A \otimes B]_{-, \sigma, \sigma^{\prime}}=i \sum_{\sigma_{2}}\left\{A_{\sigma, \sigma_{2}}, B_{\sigma 2, \sigma}\right\}$, with $\{A, B\}=\partial_{X}^{A} A \partial_{p}^{B} B-$ $\partial_{p}^{A} A \partial_{X}^{B} B$. Using these relations, we expand the kinetic equation Eq. 42 and integrate over energy to obtain the Boltzmann equation

$$
\begin{aligned}
\partial_{T} f_{\mathbf{p}, \sigma}+\nabla_{\mathbf{p}} \xi_{\mathbf{p}} \nabla_{\mathbf{R}} f_{\mathbf{p}, \sigma}-\nabla_{\mathbf{R}} U \nabla_{\mathbf{p}} f_{\mathbf{p}, \sigma}= & 2 \pi n_{i} \int \frac{d \mathbf{p}^{\prime}}{(2 \pi)^{3}}\left|v\left(\mathbf{p}-\mathbf{p}^{\prime}\right)\right|^{2} \delta\left(\xi_{\mathbf{p}}-\xi_{\mathbf{p}^{\prime}}\right)\left[f_{\mathbf{p}^{\prime}, \sigma}-f_{\mathbf{p}, \sigma}\right] \\
& +2 \pi n_{i}^{\prime} \int \frac{d \mathbf{p}^{\prime}}{(2 \pi)^{3}}\left|v^{\prime}\left(\mathbf{p}-\mathbf{p}^{\prime}\right)\right|^{2} \delta\left(\xi_{\mathbf{p}}-\xi_{\mathbf{p}^{\prime}}\right)\left[f_{\mathbf{p}^{\prime},-\sigma}-f_{\mathbf{p}, \sigma}\right] .
\end{aligned}
$$

In the one dimensional limit, Eq. 44 reduces to the Boltzmann equation Eq. 1, with the scattering coefficients related to the $q=0$ and $q=2 k_{f}$ components of the scattering potential: $r=n_{i}\left|v_{2 k_{f}}\right|^{2}$, $r_{s}=n_{i}^{\prime}\left|v_{0}\right|^{2}$, and $r_{s}^{\prime}=n_{i}^{\prime}\left|v_{2 k_{f}}^{\prime}\right|^{2}$. 


\section{References}

[1] M.N. Baibich, J.M. Broto, A. Fert, F. Nguyen Van Dau, F. Petroff, P. Etienne, G. Creuzet, A. Friederich, and J. Chazelas, Phys. Rev. Lett. 61, 2472 (1988).

[2] G. Binach, P. Grunberg, F. Saurenbach, and W. Zinn, Phys. Rev. B 39, 4828 (1989).

[3] W.P. Pratt,, Jr., S.F. Lee, J.M. Slaughter, R. Loloee, P.A. Schroeder, and J. Bass, Phys. Rev. Lett. 66, 3060 (1991); S.F. Lee, W.P. Pratt, Jr., R. Loloee, P.A. Schroeder, and J. Bass, Phys. Rev. B 46, 548 (1992).

[4] T. Valet and A. Fert, Phys. Rev. B 48, 7099 (1993).

T. Valet and A. Fert, J. Magn. Magn. Mater. 121, 378 (1993);

A. Fert, T. Valet, and J. Barnas, J. Appl. Phys. 75, 6693 (1994).

A. Fert, J.L. Duvail, and T. Valet, Phys. Rev. B 52, 6513 (1995).

[5] M.A.M. Gijs and G. Bauer, Adv. in Physics 46, 285 (1997).

[6] V.J. Falko, C.J. Lambert, and A.F. Volkov, JETP Letters 69, 532 (1999).

[7] F.J. Jedema, B.J. van Wees, B.H. Hoving, A.T. Filip, and T.M. Klapwijk, Phys. Rev. B 60, 16549 (1999).

[8] W. Belzig, A. Brataas, Yu. V. Nazarov, G.E.W. Bauer, arXiv:cond-mat/0005188.

[9] R.Y. Gu, D.Y. Xing, and Z.D. Wang, Phys. Rev. B 58, 11142 (1998).

[10] J. Chen and S. Hershfield, Phys. Rev. B 57, 1097 (1998).

[11] M. Johnson and R.H. Silsbee, Phys. Rev. B 35, 4959 (1987).

[12] P.C. Van Son, H. Van Kempen, and P. Wyder, Phys. Rev. Lett. 58, 2271 (1987).

[13] J.X. Zhu and Z.D. Wang, Phys. Rev. B 55, 8437 (1997).

[14] The Kondo Problem to Heavy Fermions, A.C. Hewson, Cambridge Studies in Magnetism, Cambridge University Press (1993).

[15] V.I. Kozub and A.M. Rudin, Phys. Rev. B 52, 7853 (1995).

[16] K.E. Nagaev, Phys. Rev. B 52, 4740 (1995).

[17] G.E. Blonder, M. Tinkham, and T.M. Klapwijk, Phys. Rev. B 25, 4515 (1982).

[18] J. Rammer and H. Smith, Rev. Mod. Physics 58, 323 (1986). 